\title{
Evolution récente de la filière cocotier en Afrique : conséquences sur la recherche agronomique tropicale
}

\section{Recent changes in the African coconut subsector and impact on tropical agriculture research}

Oléagineux, Corps Gras, Lipides. Volume 7, Numéro 2, 136-7, Mars - Avril 2000, Dossier : Afrique, plantation et développement

Auteur(s) : André ROUZIERE

Résumé : Le cocotier a été introduit en Afrique par les Portugais, qui l'ont importé d'Inde, d'abord sur la côte orientale puis sur la côte Atlantique. Cette plante exotique a été cultivée à petite échelle tout au long des côtes dans la zone intertropicale favorable, sauf en Tanzanie et au Mozambique où elle a occupé de larges superficies dès le siècle dernier. Suite au véritable " boom " qu'a connu le coprah après la Seconde Guerre mondiale, la culture s'est fortement développée sur tout le continent : création de grandes plantations privées au Mozambique, lancement de programmes nationaux de plantation en Afrique de l'Ouest (Côte d'Ivoire, Ghana). La superficie totale de cocotier en rapport s'est alors accrue, passant de 350000 à 500000 ha entre 1950 et 1970.

Summary : Coconut was introduced into Africa by the Portuguese several centuries ago, and underwent considerable development on that continent during the 20th century, notably between the $60 \mathrm{~s}$ and $80 \mathrm{~s}$. The commodity chain then stopped expanding, affected by unfavourable developments in the production context (development of lethal coconut epidemics, civil wars) and by the economic environment (stagnating copra market, liberalization, globalization). This rapid change led to strong demand for an analysis of the commodity chain, identification of new development challenges and the elaboration of appropriate solutions, causing research to redefine the content of its programmes. This resulted in the adoption of new approaches; which were less productivist and more participatory, giving a socio-economic dimension to a commodity chain type approach.

Keywords : coconut commodity chain, Africa, research-development, economics, socio-economics.

\section{ARTICLE}

\section{Évolution du contexte de la filière}

Ces grands projets de plantation s'inscrivaient dans le cadre de programmes de développement définis par les États. L'accès à la terre n'était pas contraint à cette époque, puisqu'il existait encore de vastes superficies non mises en valeur et que les autorités arbitraient facilement en faveur des projets de développement agricole, qu'ils soient de type industriel ou villageois. Cette évolution rapide s'est fortement ralentie à partir des années 80 sous l'effet de plusieurs facteurs défavorables :

- réduction progressive de la faveur accordée au cocotier au cours des trente années précédentes, sous l'effet de la compétition d'autres cultures plus rentables, comme le palmier à huile et l'hévéa, qui ont intéressé l'essentiel des programmes de plantation réalisés au cours des vingt dernières années ;

- développement d'épidémies de jaunissement mortel du cocotier dans plusieurs pays d'Afrique de 
I'Ouest (Ghana, Togo), d'Afrique centrale (Cameroun) et d'Afrique orientale (Tanzanie), entraînant l'éradication de la culture dans des pays comme le Cameroun et le Togo ;

- guerre civile au Mozambique, empêchant le développement des programmes de plantation en cours et entraînant l'abandon des cocoteraies existantes (Boror, Madal).

La libéralisation de l'économie intervenue à la fin des années 80 a encore modifié le contexte des filières cocotier africaines.

* La libéralisation a signifié l'arrêt des plans de développement décidés et mis en œuvre par l'État dans le cadre de grands projets nationaux de plantation. À noter toutefois qu'il devenait de plus en plus difficile de monter ces projets, du fait de l'accroissement des contraintes pesant sur l'accès à la terre : émergence simultanée d'une certaine pression foncière et des revendications des populations locales concernant le contrôle de l'usage de la terre.

* Sous la pression des organismes financiers internationaux, les sociétés agro-industrielles appartenant à l'État ont été privatisées, créant des rapports inédits entre les nouvelles sociétés agroindustrielles et leur environnement local (rapports avec les planteurs villageois, développement de la concurrence en amont - approvisionnement - et en aval - commercialisation des produits finaux).

* L'ensemble de ces mutations se sont déroulées dans le cadre de la globalisation de l'économie et de l'ouverture sur l'extérieur, avec des effets ambivalents : renforcement des opportunités de vente, mais aussi exposition accrue à la concurrence étrangère.

\section{Repositionnement de la recherche sur les nouveaux enjeux}

Cette évolution rapide et parfois brutale des contextes nationaux et international a eu d'importantes répercussions sur le fonctionnement des filières cocotier. Au-delà des opportunités offertes et parfois saisies (comme la compétitivité retrouvée des exportations après la dévaluation du franc CFA en Afrique francophone), ces bouleversements profonds du contexte économique ont rendu caduques les bases de raisonnement utilisées par les différents types d'acteurs intervenant dans la filière. S'en est suivie une demande forte et diversifiée de l'ensemble des acteurs et intervenants en termes d'analyse de filière, de redéfinition des enjeux, de mise au point de solutions adaptées et de développement d'outils d'aide à la décision.

La recherche agronomique a ainsi été conduite à redéfinir le contenu de ses programmes et la nature de ses interventions de façon à pouvoir répondre aux nouvelles questions se posant dans ce contexte complètement modifié. En ce qui concerne le CIRAD, l'évolution des compétences a porté en particulier sur :

- l'étude diagnostique des filières existantes, qui a donné lieu au développement d'une approche multidisciplinaire au début des années 90. Outre l'évaluation agronomique classique (potentiel de production, principales contraintes), celle-ci intègre l'analyse socio-économique des situations (identification des acteurs, de leurs objectifs et stratégies, évaluation des avantages comparatifs) et l'évaluation du potentiel de valorisation de la production (marchés, transformation). Mise au point à l'occasion de la réalisation d'études-filière réalisées entre 1993 et 1997 dans la zone Asie-Pacifique (Vanuatu, Philippines, Polynésie française), la nouvelle approche a été mise en œuvre ensuite avec succès en Afrique (études des filières cocotier du Ghana et du Mozambique) ; 
- les recherches portant sur des sujets identifiés comme prioritaires par les producteurs (sélection de matériel adapté aux conditions de culture paysannes, lutte intégrée contre les maladies mortelles, lutte biologique contre les ravageurs proliférant dans les zones d'épidémie ou vieillissantes) ;

- la mise au point d'itinéraires techniques adaptés et performants permettant d'améliorer la productivité et de sécuriser le revenu du planteur (travaux sur la réhabilitation des cocoteraies, les associations de cultures sous cocotier) ;

- l'étude de procédés innovants destinés à diversifier les usages (fabrication de nouveaux produits) et à mieux valoriser la production que sous forme de coprah ;

- le développement de modèles de simulation et d'outils prédictifs pouvant être déclinés en outils d'aide à la décision à l'usage des différents acteurs de la filière : producteurs (prédiction, aide au choix d'itinéraires techniques), négociants, transformateurs (prédiction) et responsables agricoles (prédiction, aide à l'évaluation des potentialités agricoles) ;

- la proposition d'améliorations, de réorientations, voire de solutions alternatives, concernant tant la production primaire que la transformation, la mise en marché et l'organisation globale de la filière (organisation de bassins de collecte dans le cadre d'une contractualisation entre producteurs et transformateurs).

\section{CONCLUSION}

L'évolution récente du contexte économique des pays africains a conduit la recherche à définir de nouveaux objectifs moins productivistes et plus axés sur la demande finale et sur les problèmes rencontrés par les acteurs de la filière. Ce repositionnement passe par la mise en œuvre d'approches prenant mieux en compte les réalités du terrain, les stratégies des acteurs et intégrant les différentes étapes du processus conduisant du champ à l'utilisateur final. Avec quelques années de recul, l'approche de la recherche a évolué sensiblement dans les domaines suivants :

- élargissement du champ d'intervention : ouverture de l'agronomie aux sciences sociales (socioéconomie, économie) et à la transformation ;

- diversification des représentations : du modèle unique organisé autour du nucléus agro-industriel vers la prise en compte d'ensembles plus complexes faisant co-exister plusieurs systèmes de production et de transformation ;

- adoption d'une approche participative, associant étroitement les producteurs à la sélection des problèmes prioritaires, à l'identification de solutions adaptées et à leur validation en conditions réelles ;

- adoption d'une démarche filière intégrant les différentes étapes du processus conduisant du champ au consommateur pour une meilleure prise en compte de la demande du marché.

Les quelques articles figurant dans ce dossier « replantation » illustrent bien l'évolution récente de la recherche agronomique tropicale. 